\title{
A Sensor-augmented Belt toward Abdominal Shape Measurement System
}

\author{
Yukihiro Oishi, Airi Tsuji, and Kaori Fujinami \\ Department of Computer and Information Sciences, Tokyo University of Agriculture and Technology, \\ 2-24-16 Naka-cho, Koganei-shi, Tokyo 184-8588, Japan
}

(Received October 26, 2021; accepted November 30, 2021)

Keywords: belt-type device, bending sensor, girth shape, shape estimation

In this study, we developed a system for measuring the shape of a wearer's abdominal circumference using a belt-type wearable device for the daily assessment of metabolic syndrome. The force applied to the belt from the abdomen was estimated using force sensors, and the shape of the abdominal circumference when the belt was not worn was estimated by considering the effect of outward force on the belt shape estimation. Using the developed device, we conducted evaluation experiments on eight adult males: two with a thin body type (BMI < 18.5), four with a standard body type $(18.5 \leq \mathrm{BMI}<25)$, and two with an overweight body type $(25 \leq \mathrm{BMI})$. The results showed that it was possible to estimate the shape of the nonwearing abdominal circumference with an average error of $3.73 \%$. As a result of comparing the sensor values in the reference situation and the state in which each state changed, it was clarified that the system could detect the upright and exhale states appropriate for measurement.

\section{Introduction}

The increase in the number of obese patients has become a serious social problem in Japan. According to a report by the Japanese Ministry of Health, Labour and Welfare (MHLW), approximately $30 \%$ of adult males and $20 \%$ of adult females are overweight. ${ }^{(1)}$ Obesity is one of the factors that increase the risk of dyslipidemia, glycosuria, hypertension, and atherosclerosis. ${ }^{(2)}$ In addition, obesity, when combined with hypertension, hyperglycemia, and abnormal lipid metabolism, leads to metabolic syndrome, which is an important risk factor for the development of cardiovascular diseases such as stroke and heart disease. ${ }^{(3)}$

It has been reported that the incidence of metabolic syndrome is 1.5 times higher when the visceral fat area is $100 \mathrm{~cm}^{2}$ or greater. ${ }^{(4)}$ For this reason, the visceral fat area is used as a diagnostic criterion for metabolic syndrome. However, the measurement of the visceral fat area is not easy because it requires special techniques, such as computer tomography (CT) or magnetic resonance imaging (MRI), and specialized technicians to handle them. Therefore, a simple screening method that focuses on the abdominal circumference, $85 \mathrm{~cm}$ for male and 90 $\mathrm{cm}$ for female as reference values, is used in periodic health examinations for the early detection

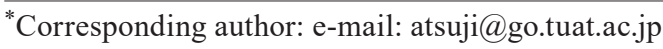
https://doi.org/10.18494/SAM.2021.3715 
of metabolic syndrome and guidance on lifestyle improvement for patients and pre-metabolic syndrome groups. However, there are some cases of hypertrophy that cannot be detected by only measuring abdominal circumference, and it has been reported that the use of abdominal crosssectional area and abdominal circumference shape, in addition to abdominal circumference, is effective in estimating visceral fat mass. ${ }^{(5)}$ There are various methods of measuring the shape of the abdominal circumference, such as measuring the visceral fat area by X-ray CT and the crosssectional shape of the abdomen by 3D scanning; however, these methods have problems, such as large equipment and high cost, and hence, a system that can easily measure the shape of the abdominal circumference is required. In this study, we propose a belt-type device that estimates the shape of the abdominal circumference in such states as standing, static, and exhale. The device measures the shape of the abdominal circumference and the user's situation with bending and force sensors more easily than by using large equipment.

The remainder of this article is organized as follows. In Sect. 2, related works are introduced to show the importance of our research. Section 3 shows an overview of the proposed system and belt-type device. Also, a sensor calibration method and its results are introduced. Section 4 shows the results and analysis of the experiments performed to measure the shape of the abdominal circumference using the developed device. The results and analysis of the experiments performed to estimate the user's situation are discussed in Sect. 5. Section 6 presents our conclusions. Note that the experimental protocol used was approved by the Institutional Review Board of Tokyo University of Agriculture and Technology (\#201204-0265).

\section{Related Works}

Although there are methods of using large equipment such as CT and MRI to obtain the shape of the abdominal circumference, these methods have problems in terms of invasiveness and cost, and measuring the circumference shape on a regular basis is difficult. Therefore, research has been conducted to measure the shape of the human abdominal circumference easily and at a low cost. Watanabe et al. ${ }^{(6)}$ have developed a system for measuring abdominal girth and cross-sectional area using a laser displacement meter. In addition, a study to estimate the visceral fat area from the cross-sectional shape of the abdomen has been conducted. ${ }^{(5)}$ Nkwana et al. used a rangefinder to measure the distance between the device and the abdomen, and the threedimensional shape of the abdomen was obtained. ${ }^{(5)}$

As research on shape acquisition by devices, CheekInput ${ }^{(7)}$ detects the shape of a cheek by placing multiple optical sensors inside glasses. This system recognizes the shape of the cheek by measuring the distance from the frame of the glasses to the cheek using optical sensors and by learning the cheek shape from the touch gesture. In addition, Williams et al. ${ }^{(8)}$ and Dunne et al. ${ }^{(9)}$ proposed a method of measuring the shape of the spine by attaching an optical fiber sensor along the spine. For measuring the shape of the device itself, FlexiBend ${ }^{(10)}$ developed by Chien et al. can be used. This device consists of strain gages arranged in a linear pattern and can measure the shape of the object itself.

Belt-type devices can be divided into two types: output devices and measurement devices. As an output device, Tsukada and Yasumura proposed a personal navigation system ActiveBelt ${ }^{(11)}$. 
This system obtains the current location using a geomagnetic sensor installed on the belt and GPS information and guides the user to the destination by driving a vibration motor in the guidance direction. Katzschmann et al. ${ }^{(12)}$ proposed a navigation belt with haptic feedback that combines an array of time-of-flight distance sensors and a vibration device. As a measurement device, Cajamarca et al. ${ }^{(13)}$ installed three accelerometers on a harness belt for monitoring the seated spinal posture. The belt used the values obtained via sensors to identify the posture using a classification model based on a decision tree. In addition, $\mathrm{Wu}$ et al. ${ }^{(14)}$ achieved an overall identification accuracy of $93 \%$ in basic actions such as standing, jumping, sitting, and walking in action classification using a single accelerometer installed in a waist belt. Matsuda et al. proposed the use of WaistonBelt $2^{(15)}$ to measure the abdominal circumference. This system uses photoreceptors in the belt buckle and measures the abdominal circumference by estimating the insertion distance of the belt by counting the number of pulses obtained from a rotary encoder. Using the acceleration sensor attached to the buckle, we have shown the possibility of classifying the wearer's posture and eating behavior. Furthermore, the possibility of estimating the shape of the abdominal circumference by measuring the tension of the abdomen using force-sensitive sensor data is also described.

There have been numerous studies on activity recognition by wearable devices. For example, Zhang et al. designed an embedded device that can be worn on fingers. ${ }^{(16)}$ By finger clicking or sliding on any surface, one can control a screen from any distance, just like using a touchpad. There is a study on recognizing the shape of a finger using a ring-shaped device. ${ }^{(17)}$ In this research, the bending of the finger is recognized by considering the fact that the fingertip approaches the device when the finger is bent, whereby the capacitance increases. There is also a study on estimating the posture of the user by attaching accelerometers to multiple positions on the body. ${ }^{(13,18)}$ In this manner, several studies have been conducted to recognize the posture and behavior of a wearer using a wearable sensor device.

Among wearable devices, a belt-type device that can be worn around the abdomen is suitable for measuring the shape of the abdominal circumference. Since the shape of the abdominal circumference changes depending on the wearer's posture and behavior, the wearer's state should be estimated. To estimate the state of the wearer, attaching a sensor to the waist ${ }^{(19-20)}$ is effective. Therefore, we estimate the state of the wearer by using a belt-type device that can acquire data from the waist. In addition, since belts are often used in daily life, they have the advantage of not interfering with the daily activities of the wearer.

\section{Abdominal Circumference Shape Measurement System}

\subsection{Application scenario}

As a scenario for using the proposed system, we assume that the system automatically starts measurement when users put on the belt-type device after waking up. As users wear the device as they go about their daily life, the system estimates the users' state and detects when the users are in the reference situation (standing, static, and exhale) as a suitable means to measure the abdominal circumference shape, ${ }^{(15,21)}$ such as when users are waiting at a traffic light or standing 
in a train, and estimates and records the abdominal circumference shape at that time. When users return home, they can check the shape of their abdominal circumference for the day on their smartphone and confirm the degree of obesity. Furthermore, on weekends, users can compare their current abdominal circumference with that of the previous week to reflect on their weekly lifestyle and hence manage their health to prevent lifestyle-related diseases. In this manner, this system detects the timing close to the users' reference situation and estimates the abdominal circumference shape of the users wearing it. The system can warn users of obesity and promote health management by informing them of the history of their abdominal circumference.

\subsection{Overall system design}

As shown in the above scenario, the system is realized as a wearable system, in which a belt is augmented with sensing capabilities to estimate the shape of the abdominal circumference. In addition, the system is more practical if it automatically determines the timing close to the reference situation, rather than asking the wearer to recall and input the reference timing or to be in the reference state. The automatic detection of the reference situation allows the wearer to act normally. In this study, we aim to detect the timing when the wearer's situation is close to the reference situation and estimate the shape of the abdominal circumference at that timing. The outline of the proposed system is shown in Fig. 1, which is described in this section.

As for the accuracy of the estimation of the shape of the abdominal circumference, research on the measurement of the shape of the abdominal circumference using an external device ${ }^{(6)}$ has shown that measuring the shape of the abdominal circumference with a maximum error of $3.4 \%$ using a laser displacement meter is possible. In this study, we aim to achieve an estimation error of $3.4 \%$ or less using only the wearable device and define it as an acceptable error.

The shape of the belt is approximated as an ellipse, and the lengths of the semi-major and semi-minor axes are subject to estimation. A point $(x, y)$ on the ellipse is represented by Eqs. (1)

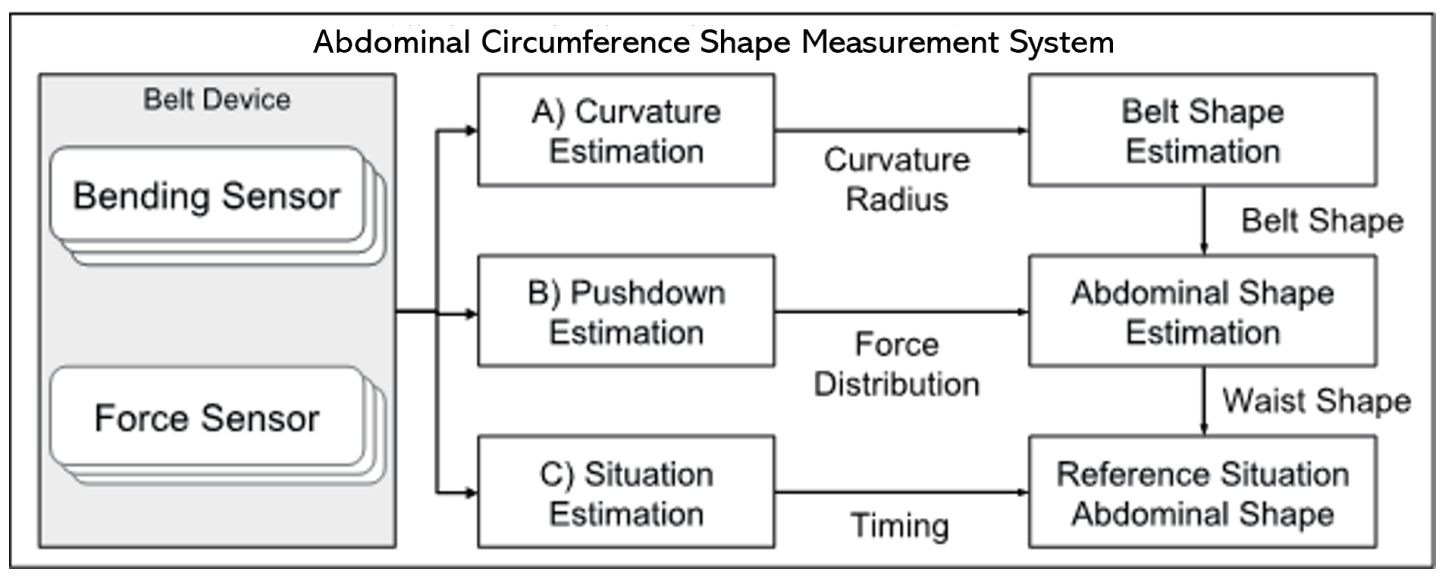

Fig. 1. Major components of abdominal circumference shape measurement system. 
and (2) using an arbitrary angle $\theta$ as a mediator variable, where $a$ and $b(0<b<a)$ represent the semi-major and semi-minor axes, respectively.

$$
\begin{gathered}
x=x(\theta)=a \cos \theta \\
y=y(\theta)=b \sin \theta \\
R(\theta)=\frac{\left(x^{2}+y^{2}\right)^{3 / 2}}{\left|x^{\prime} y^{\prime \prime}-y^{\prime} x^{\prime \prime}\right|}
\end{gathered}
$$

The radius of curvature $R(\theta)$ is expressed by Eq. (3), where $x^{\prime}=\mathrm{d} x / \mathrm{d} \theta$ and $y^{\prime}=\mathrm{d} y / \mathrm{d} \theta$.

Then, by substituting Eqs. (1) and (2) into Eq. (3), the relationship between the radius of curvature and the elliptic radius is represented by Eq. (4), where $a$ and $b$ are derived using the curvatures $R\left(\theta_{1}\right)$ and $R\left(\theta_{2}\right)$ obtained for the two angles $\theta_{1}$ and $\theta_{2}$, respectively. Curvature estimation (Fig. 1A) enables this calculation.

$$
R(\theta)=\frac{\left(a^{2} \sin ^{2}(\theta)+b^{2} \cos ^{2}(\theta)\right)^{3 / 2}}{a b}
$$

The belt shape is not approximated by a single ellipse, rather by two ellipses representing the front and back parts of the belt.

The ideal shape of the abdominal circumference, i.e., the situation in which the belt is not worn, might, however, differ from the shape of the belt because the abdomen is tightened by the belt. We consider that the distribution force applied to the belt from the abdomen can be used to estimate the ideal shape while constricting the abdomen. Pushdown estimation (Fig. 1B) is performed to calculate the force using force-sensitive sensors. The ideal shape is estimated by adding the force to the outside of the belt shape.

In addition, since the shape of the abdominal circumference changes depending on the situation of the wearer, ${ }^{(15,21)}$ the measurement should be performed at an appropriate timing, i.e., a situation close to the reference situation. Thus, the state estimation component (Fig. 1C) plays a role in determining the appropriate timing. This component is realized using the same sensors as in the belt shape and abdominal shape estimations, i.e., a bending sensor and a force sensor, so that the number of sensors can be reduced to make the system practical and wearable.

\subsection{Implementation of the belt-type device}

The position of the sensors on the belt relative to the torso is strongly affected by the size of the abdominal circumference. In other words, if a sensor is fixed to fit a user with a small 
abdominal circumference, its position will be unsuitable for a user with a large abdominal circumference. Moving the sensor for each abdominal circumference size increases the complexity of the system. Therefore, the sensor positions are concentrated on the left side, as shown in Fig. 2, to reduce the error. Seven bending sensors and seven force sensors were installed on the belt at the left side of the wearer.

The bending sensors were placed at $165,170,175,180,185,190$, and $195^{\circ}$, with the right side of the wearer being $0^{\circ}$. This is because the radius of curvature increases when the position is closer to the front or back of the wearer, and measuring the radius of curvature using the bending sensor is difficult. In addition, considering the belt width and sensor size, the sensors were placed at $5^{\circ}$ intervals, resulting in seven positions. One of the two angles $\left(\theta_{1}\right.$ and $\left.\theta_{2}\right)$ for the front part is chosen from among the three angles corresponding to the three sensors installed at the front part (in Fig. 2) of the belt $\left(165,170\right.$, and $\left.175^{\circ}\right)$, while the other is that of the sensor located at the side part $\left(180^{\circ}\right)$. Similarly, one of the two angles for the back part is chosen from among those of the three sensors at the back part $(185,190$, and 195 $)$ in Fig. 2 and the other is that of the sensor at the side part. The combination of the two sensors is evaluated in Sect. 4.

The force sensors were placed at $150,165,180,195,210,225$, and $240^{\circ}$. We ignored the angles between 90 and $135^{\circ}$ because it was difficult to measure the abdominal pressure when the force sensors were close to the front part of the buckle. By contrast, the angles between 255 and $270^{\circ}$ were not used because the spine made it difficult to measure the amount of pushdown accurately. The force sensors were placed $15^{\circ}$ apart from each other, because it would be difficult to measure the force on the belt when the sensors are very close to each other.

Figure 3 shows the prototype belt-type device in our actual evaluation. The raw data from the bending sensors (Spectra Symbol FS-L-0055-253-ST) ${ }^{(22)}$ and force sensors (ALPHA MF01-N221-A01) ${ }^{(23)}$ were transmitted to a personal computer (PC) via Arduino Mega, and the estimation processes were performed. Both the bending and force sensors are variable resistors and Arduino measures the potential difference between the ends of the pull-down resistors. This means that the sensor values increase as the curvature radius decreases and the force applied increases in the bending and force sensors, respectively. The integration of all the functionalities into the belt is considered to be a task in our future work.

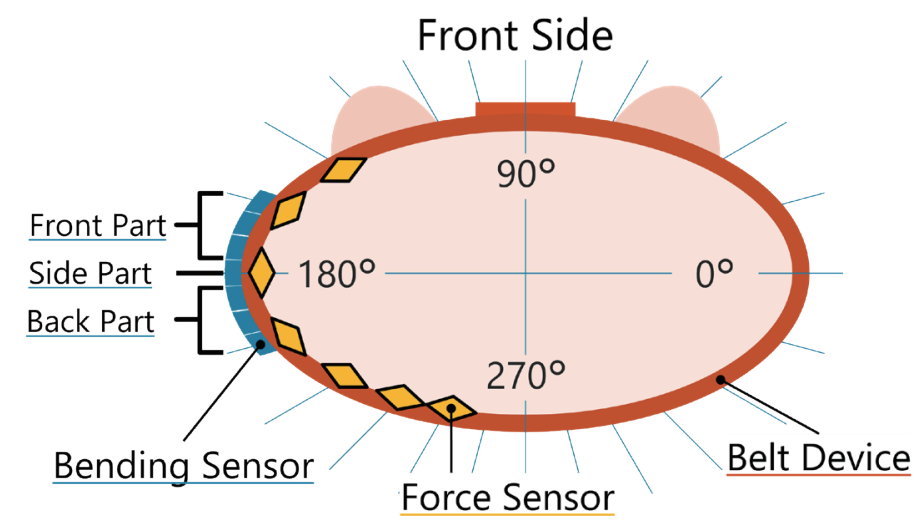

Fig. 2. (Color online) Sensor placement on belt device. 


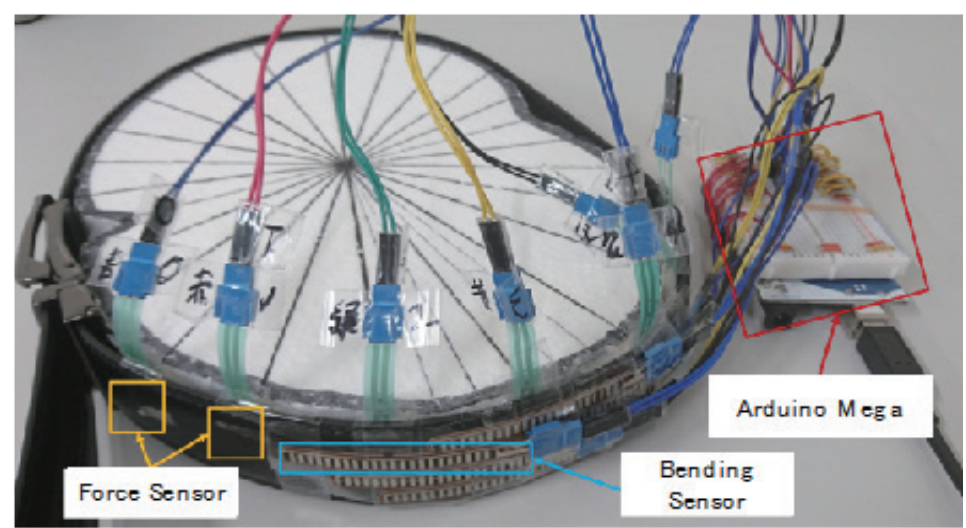

Fig. 3. (Color online) Prototype of belt-type device with Styrofoam.

The resistance in the straight-line state $\left(R_{B}\right)$ is obtained using Eq. (5) from the bending sensor value in the straight shape, where $x$ is the raw sensor value quantized at 10 bits and $R_{1}$ is the resistance of the pull-down resistor.

$$
R_{B}=\frac{1024 \times R_{1}}{x}-R_{1}
$$

The bending sensor only outputs the resistance in accordance with the bending force, not the radius of curvature. Therefore, the user should know the relationship between the resistance and the radius of curvature. To account for this individual variation, the relationship between the sensor value and the radius of curvature for each sensor should be investigated. In this study, we investigated a calibration tool by cutting Styrofoam (thickness: $30 \mathrm{~mm}$ ) into 60 to $120 \mathrm{~mm}$ pieces for each $20 \mathrm{~mm}$ radius, as shown in Fig. 4. With the calibration tool, we measured the bending sensor values when the sensor was bent to a certain radius of curvature and modeled the relationship between each sensor value and the radius of curvature using cubic spline interpolation (Fig. 5). Using this relationship, we estimated the radius of curvature $[R(\theta)$ in Eqs. (3) and (4)] from the sensor value. The radius of curvature is estimated from the front and side bending sensor values and the front part of the belt shape is estimated as an ellipse using Eq. (4).

The ideal shape of the abdominal circumference is determined by considering the distribution of the force applied to the belt from the abdomen, in order to estimate the difference in the distance to the center of the body shrunken by the belt. Since the force sensors are installed only at $15^{\circ}$ intervals in the range of 150 to $240^{\circ}$, the values of the other positions should be estimated using "virtual" sensors before estimating the difference in the distance. Assuming that the body is symmetrical, the values of the virtual sensors on the left side of the body are estimated first, and the right-side values correspond to the left-side values. The values of the virtual sensors in the front part, i.e., $135,120,105$, and $90^{\circ}$, are obtained using the values of the two nearest "real" sensors, i.e., 165 and $150^{\circ}$, by extrapolation. The value of a particular virtual sensor is calculated in a manner similar to the external division. For example, the sensor at $135^{\circ}$ is considered to be 

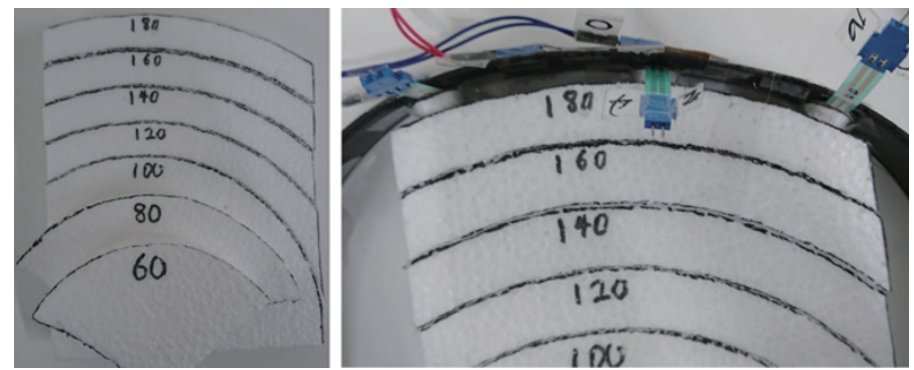

Fig. 4. (Color online) Bending sensor calibration.

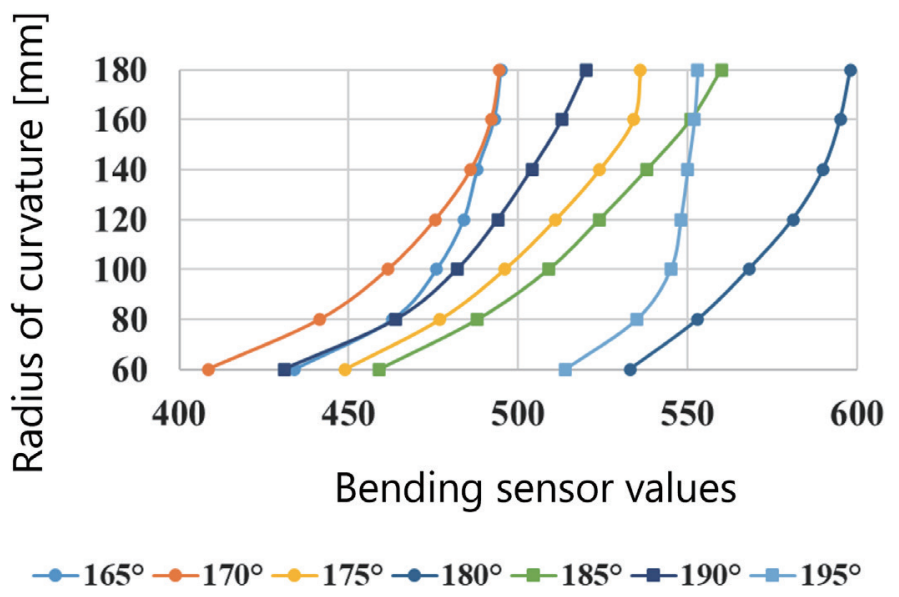

Fig. 5. (Color online) Relationship between bending sensor values and radius of curvature.

externally dividing the sensors at 165 and $150^{\circ}$ to $2: 1$. Let the label of the force sensor placed at an angle of $\theta$ and its value be $F_{\theta}$ and $V_{F}(\theta)$, respectively. The value of a virtual sensor $F_{Q}$ is represented by Eq. (6), where $Q=150-15 \times n$ and $n \in\{1,2,3,4\}$. The equation is simplified by using the relation $m-n=1$, indicating that F165 and F150 are close to each other [Eq. (7)]. Thus, for example, $V_{F}(135)$ and $V_{F}(90)$ are represented by $-F_{165}+2 \cdot F_{150}$ and $-4 \cdot F_{165}+5 \cdot F_{150}$, respectively.

$$
\begin{aligned}
V_{F}(Q) & =\frac{-n \cdot F_{165}+m \cdot F_{150}}{m-n} \\
& =-n \cdot F_{165}+(n+1) F_{150}
\end{aligned}
$$

The values of the virtual sensors in the back part are estimated differently. The value of the virtual sensor at an angle of $270^{\circ}\left[V_{F}(270)\right]$ is assumed to be zero because there is not enough fat near the spine to generate force to press the force sensor, if any. Similarly, $V_{F}(255)$ is obtained by setting half of the value of the adjacent sensor, i.e., $0.5 \cdot V_{F}(240)$. 
To obtain the displacement along the center of the body, the relationship between the force applied to the force sensor from the body fat and the sensor value should be modeled. Thus, a plug with a height of 5,10 , or $15 \mathrm{~mm}$ was attached to the center of the force sensor calibrator (Fig. 6), and the calibrator was pressed against the abdomen to obtain the pressure-sensitive sensor values at each height. Then, the cubic spline interpolation was used for modeling, as shown on the left side of Fig. 7. Finally, the pressure or displacement, at each sensor position $(Q)$ was estimated using the relationship shown on the right side of Fig. 7 by applying $V_{F}(Q)$ as an input. Here, we consider the displacement as $D_{F}(Q)$, which is drawn by a red arrow on the right side of Fig. 8. The actual abdominal circumference $\left[D_{A}(Q)\right]$ is then obtained by extending a straight line outward from the origin (the center of the body) at an angle $Q$ by an additional length $D_{F}(Q)$ from the point of intersection with the ellipse representing the belt shape [Eq. (8)]. Note that the distance from the origin to the intersection of the ellipse at $Q$ is represented by $D_{B}(Q)$. The two-dimensional plot can be drawn by converting the polar coordinates $(D, Q)$ into Cartesian coordinates.

$$
D_{A}(Q)=D_{B}(Q)+D_{F}(Q)
$$

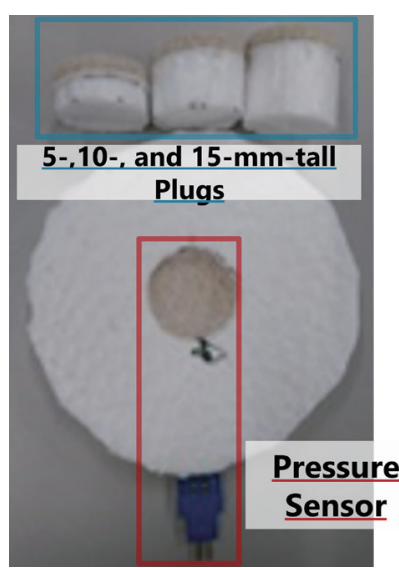

Fig. 6. (Color online) Force sensor calibrator and plugs of different heights.
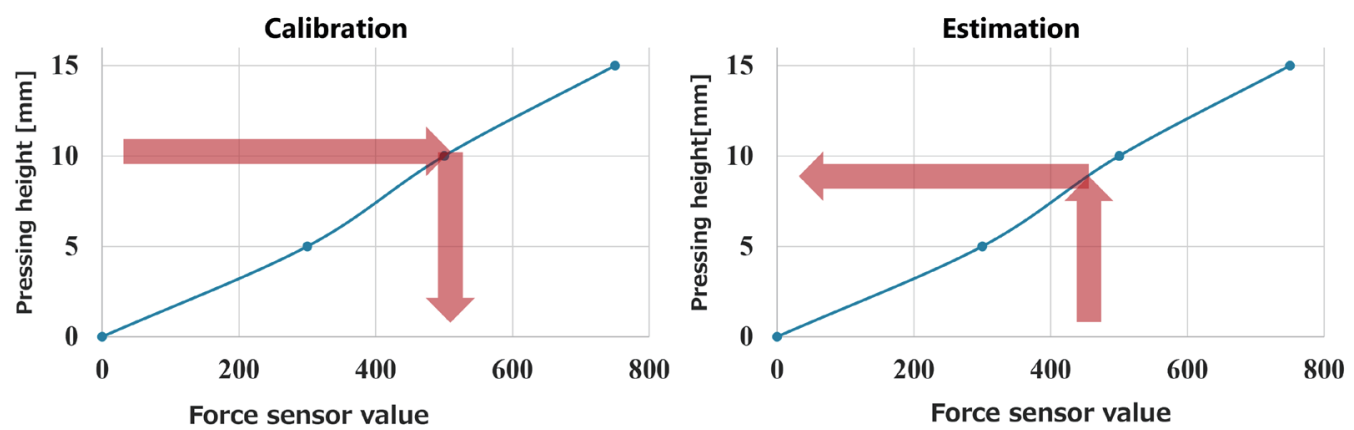

Fig. 7. (Color online) Relationship of force sensor value and pressing height. 


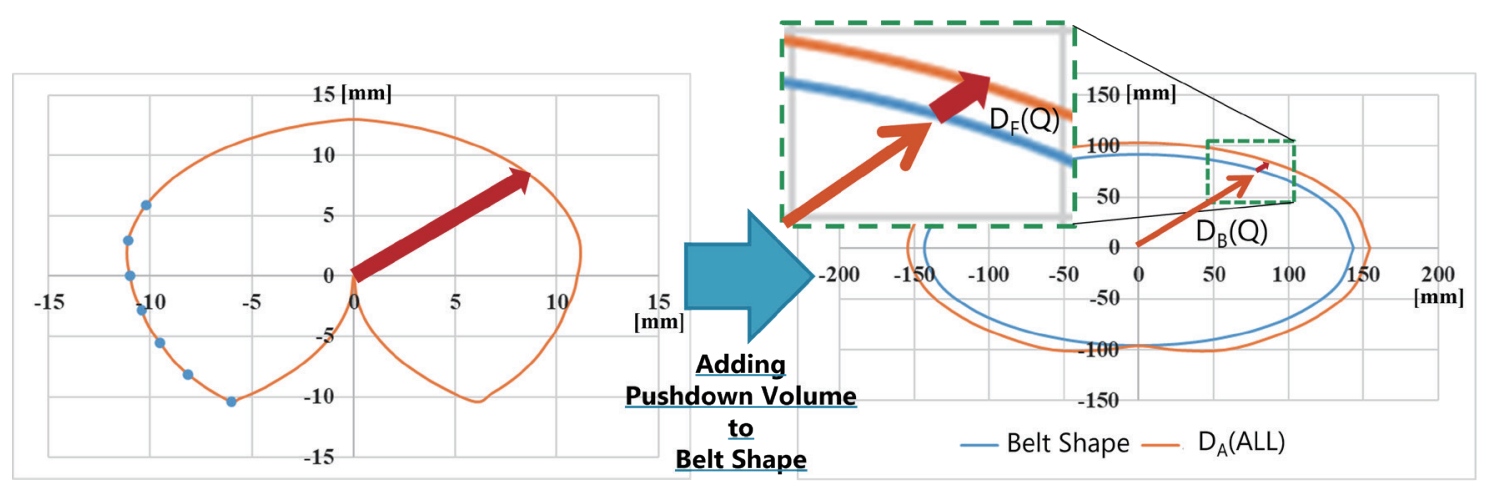

Fig. 8. (Color online) Estimation of abdominal shape using pushdown volume.

\section{Evaluation of Appropriate Sensor Position}

Two experiments were conducted to verify the effectiveness of the proposed system. The first experiment was intended to identify the effective sensor positions for estimating the shape of the abdominal circumference. The purpose of this experiment was to measure the shape of the abdominal circumference, and since it is known that the estimation error differs depending on the body shape of the wearer, ${ }^{(21)}$ investigation of various abdominal circumferences is required. The estimation error may vary depending on the body shape of the wearer. Therefore, we will clarify the appropriate sensor position for each body type of the user.

\subsection{Method}

The experiment was conducted on eight male university students in their early twenties. Therefore, on the basis of the BMI classification defined by the Japan Society for the Study of Obesity, various body types from thin to overweight were targeted. Table 1 shows the body type and BMI of each subject.

To evaluate the estimation results, a ground truth is necessary. In this study, we define the ground truth as the values measured using a dedicated tool. For this purpose, we developed a tool consisting of 24 rulers that move toward the center of the body as shown in Fig. 9. The method of using the measuring tool and the measurement accuracy are described in Sect. 4.2.

The subjects wore the belt-type device, and the sensor values in the reference situations (standing, static, and exhale) were obtained. The shape of the abdominal circumference was estimated in the reference situations using these sensor values, the estimation accuracy was evaluated by comparing the ground truth, and the values were estimated using the belt-type device. We evaluated the effects of various combinations of bending sensors in different positions in terms of the estimation accuracy. As described in Sect. 3.3, in the estimation of the entire abdominal circumference, one sensor from the side part and one from the front part are used to estimate the first half, and one from the side part and one from the back part are used to estimate the second half shown in Fig. 2. Thus, there are three ways to estimate the first half and 
Table 1

BMI of subjects.

\begin{tabular}{lcc}
\hline Body Type & Subjects & BMI \\
\hline \multirow{2}{*}{ Thin $(\mathrm{BMI}<18.5)$} & $\mathrm{A}$ & 17.0 \\
\hline & $\mathrm{B}$ & 18.2 \\
\cline { 2 - 3 } Standard $(18.5 \leq \mathrm{BMI}<25)$ & $\mathrm{C}$ & 20.6 \\
\cline { 2 - 3 } & $\mathrm{D}$ & 20.9 \\
\cline { 2 - 3 } & $\mathrm{E}$ & 21.8 \\
\hline \multirow{2}{*}{ Overweight $(25 \leq \mathrm{BMI})$} & $\mathrm{F}$ & 22.5 \\
\hline
\end{tabular}

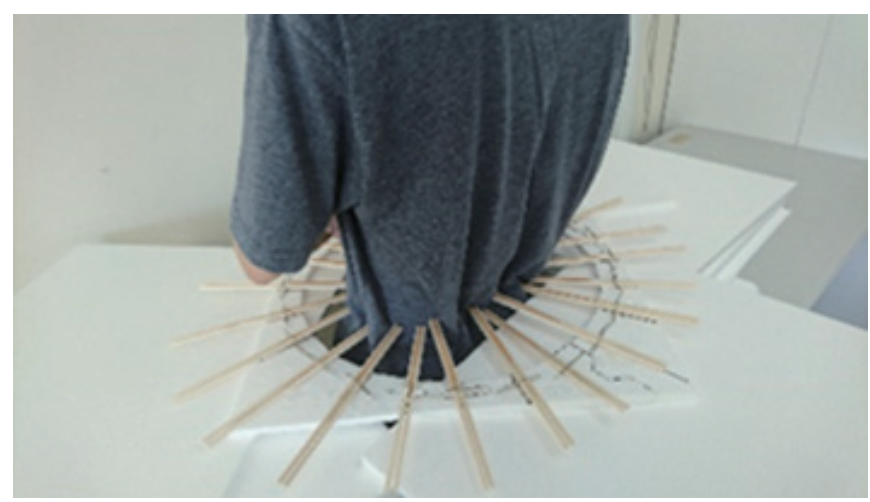

Fig. 9. (Color online) Using the measuring tool.

three ways to estimate the second half, resulting in a total of nine ways. Therefore, the mean absolute errors (MAEs) are obtained for the total of nine ways. In this analysis, all force sensors are first used to estimate the force distribution. We calculated the estimation error for $2^{7}=128$ combinations by enabling/disabling each of the seven force sensors on the device. Next, we obtained the average of the estimation errors calculated for each subject for each combination of force sensors. For each number of sensors used, we identified the combination with the smallest estimation error and evaluated the relationship between the number of sensors and the estimation error.

Equation (9) represents the relative error $E_{i}$ for each $15^{\circ}$, where $i$ is the index of the angle and $V_{E}$ and $V_{M}$ represent the estimated value and the measured value (ground truth), respectively. MAE is defined by Eq. (10). Finally, we estimate the belt shape using the combination of bending sensors with the smallest MAE for each subject for comparison with the result obtained using the measuring tool.

$$
\begin{gathered}
E_{i}=\frac{V_{E}-V_{M}}{V_{M}} \times 100 \\
M A E=\frac{\sum_{\mathrm{i}=0}^{23}\left|E_{i}\right|}{24}
\end{gathered}
$$




\subsection{Measuring tool}

To obtain the ground truth by measuring the shape of the subject's abdominal circumference, a measuring tool consisting of a Styrofoam base with thin rods placed every $15^{\circ}$ was developed. Figure 9 shows the scene of using the measuring tool. First, the measuring tool is placed on the object to be measured. Next, the thin rod is moved toward the center of the object until it touches the object. Then, the measuring tool is removed from the object, and the length $d$ is measured from a reference line drawn $20 \mathrm{~cm}$ from the center to the tip of each stick. Finally, the length in each direction is calculated to obtain the position of the object to be measured every $15^{\circ}$.

\subsection{Results and analysis}

\subsubsection{Combination of bending sensor according to body shape}

Table 2 shows the MAE of the combination of bending sensors for subjects $\mathrm{C}, \mathrm{D}, \mathrm{E}$, and $\mathrm{F}$ with the standard body shape. As a result of the experiment, we found that the smallest MAE (2.97\%) was obtained for the combination of $170^{\circ}$ (front) and $190^{\circ}$ (back) bending sensors. In addition, we found that the MAE of the combination of $175^{\circ}$ and $185^{\circ}$ bending sensors was larger than those of the other combinations. This may be because the accuracy of estimation decreases when measuring a shape with a large radius of curvature owing to the characteristics of the bending sensor used in this experiment. For this reason, the combination of $170^{\circ}$ and $190^{\circ}$ bending sensors, which have smaller radii of curvature, reduced the MAE in this experiment. In Table 3, the effectiveness of the combinations of bending sensors for thin subjects A and B is shown. The acceptable error of $3.4 \%$ could not be achieved even with the best combination of bending sensors ( $4.65 \%$ with the combination of 160 and $195^{\circ}$ bending sensors). We consider that this is due to the characteristics of our estimation method. In this system, the belt shape is estimated using two bending sensors, including the side sensors, in the front and back sections. In our system, the belt shape is estimated by using two bending sensors including the side part in each of the front and back parts. The problem with this method is that if a bending sensor that is close to the side part is used, the error becomes larger unless the estimation is more accurate. In contrast, we found that the bending sensors that were not effective were those at $175^{\circ}$ in the front part and $185^{\circ}$ in the back part, similar to the results of the experiment with standard-body subjects. Finally, the experimental results for overweight subjects $\mathrm{G}$ and $\mathrm{H}$ are shown in Table 4. We found that the most effective sensor position for estimating abdominal circumference was the combination of 170 and $195^{\circ}$ bending sensors at the front and back parts, respectively; however, even the best combination had a large MAE of $6.2 \%$. This was because the belt shape of overweight subjects deviated greatly from the elliptical shape. The bending sensors at $175^{\circ}$ in the front part or $185^{\circ}$ in the back part for any body shape are ineffective.

\subsubsection{Placement of force sensors}

Figure 10 shows the change in estimation error with the number of force sensors used for subjects with the standard body shape. We aim to achieve an acceptable error of $3.4 \%$ or less 
Table 2

MAEs of standard-body subjects (underline means the best combination).

\begin{tabular}{ccccc}
\hline \multicolumn{2}{c}{$\begin{array}{c}\text { Standard-body subjects } \\
\text { MAE (\%) }\end{array}$} & \multicolumn{3}{c}{ Back part } \\
\cline { 2 - 5 } Front Part & $165^{\circ}$ & $195^{\circ}$ & $190^{\circ}$ & $185^{\circ}$ \\
\cline { 2 - 5 } & $170^{\circ}$ & 3.76 & 3.27 & 15.88 \\
\cline { 2 - 5 } & $175^{\circ}$ & 3.30 & 2.97 & 15.39 \\
\hline
\end{tabular}

Table 3

MAEs of thin-body subjects (underline means the best combination).

\begin{tabular}{llccc}
\hline \multirow{2}{*}{$\begin{array}{c}\text { Thin-body subjects } \\
\text { MAE (\%) }\end{array}$} & & \multicolumn{3}{c}{ Back part } \\
\cline { 3 - 5 } Front Part & $165^{\circ}$ & $195^{\circ}$ & $190^{\circ}$ & $185^{\circ}$ \\
\cline { 2 - 5 } & $170^{\circ}$ & $\underline{4.65}$ & 5.16 & 11.09 \\
\cline { 2 - 5 } & $175^{\circ}$ & 4.80 & 5.73 & 11.20 \\
\hline
\end{tabular}

Table 4

MAEs of overweight-body subjects (underline means the best combination).

\begin{tabular}{lcccc}
\hline \multicolumn{2}{c}{$\begin{array}{c}\text { Overweight-body subjects } \\
\text { MAE [\%] }\end{array}$} & \multicolumn{3}{c}{ Back Part } \\
\cline { 3 - 5 } Front Part & $165^{\circ}$ & $195^{\circ}$ & $190^{\circ}$ & $185^{\circ}$ \\
\cline { 2 - 5 } & $170^{\circ}$ & 8.25 & 8.61 & 15.45 \\
\cline { 2 - 5 } & $175^{\circ}$ & $\underline{6.20}$ & 7.92 & 16.64 \\
\hline
\end{tabular}
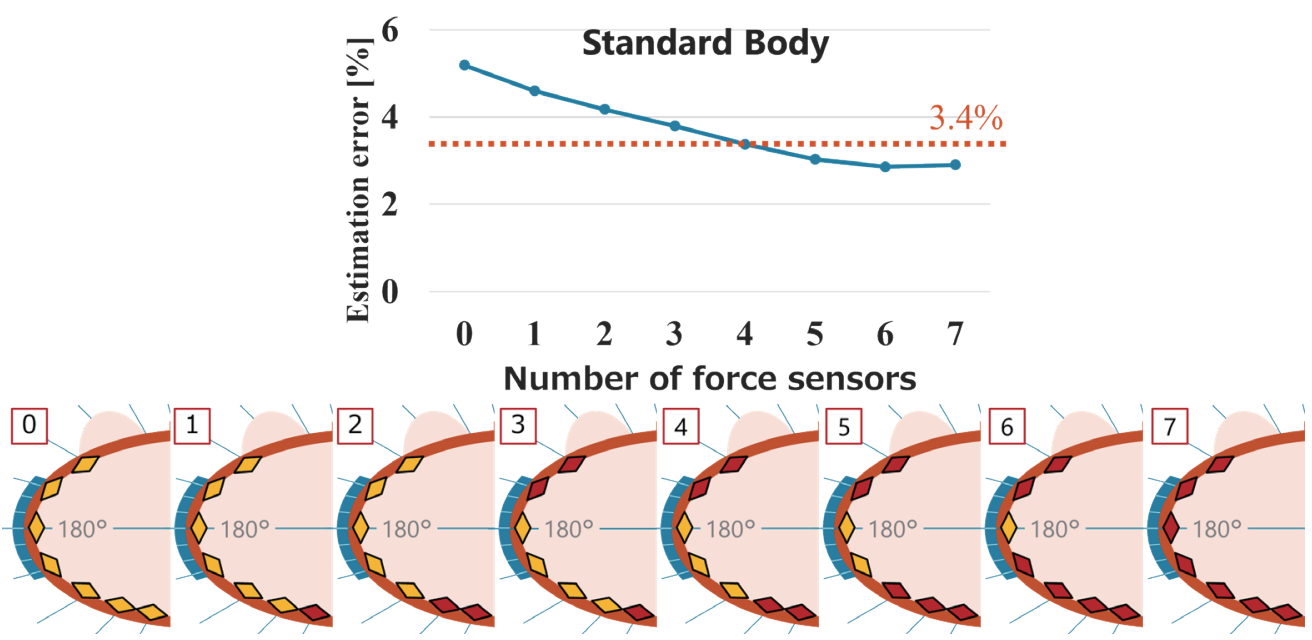

Fig. 10. (Color online) Average error of standard body vs number of force sensors.

using only the wearable device, as mentioned in Sect. 3.2. The upper figure shows the change in error with the number of force sensors used, while the lower figure shows the combinations with a smaller error for each number of force sensors. The estimation error was reduced by using the force sensor on the back side when the measurement uses as few sensors as possible. When four or more force sensors are used, the shape of the abdominal circumference can be estimated more accurately by using the frontal force sensor. 
The results of the effectiveness of the force sensors on thin subjects are shown in Fig. 11. These results showed that the estimation error became smaller than the acceptable error of $3.4 \%$ when the force sensor at the back of the body was used. This is because the force sensor measures the force on the abdominal fat applied by the belt, but subjects with a thin body shape have less fat, so the effect of the force sensor measurement was small. Also, since subjects with a thin body shape had little fat in the front area, the effectiveness of the force sensor in the front area also became low. Therefore, reducing the estimation error to below $3.4 \%$ is difficult even if the number of force sensors is increased. In addition, the abdominal circumference of the thin body type was smaller than the belt shape owing to less fat. Therefore, the estimation of the abdominal circumference inside the belt shape is required.

For overweight subjects, the estimation error was larger than those of the other body types (Fig. 12); however, the estimation error decreased with increasing number of sensors. In addition,

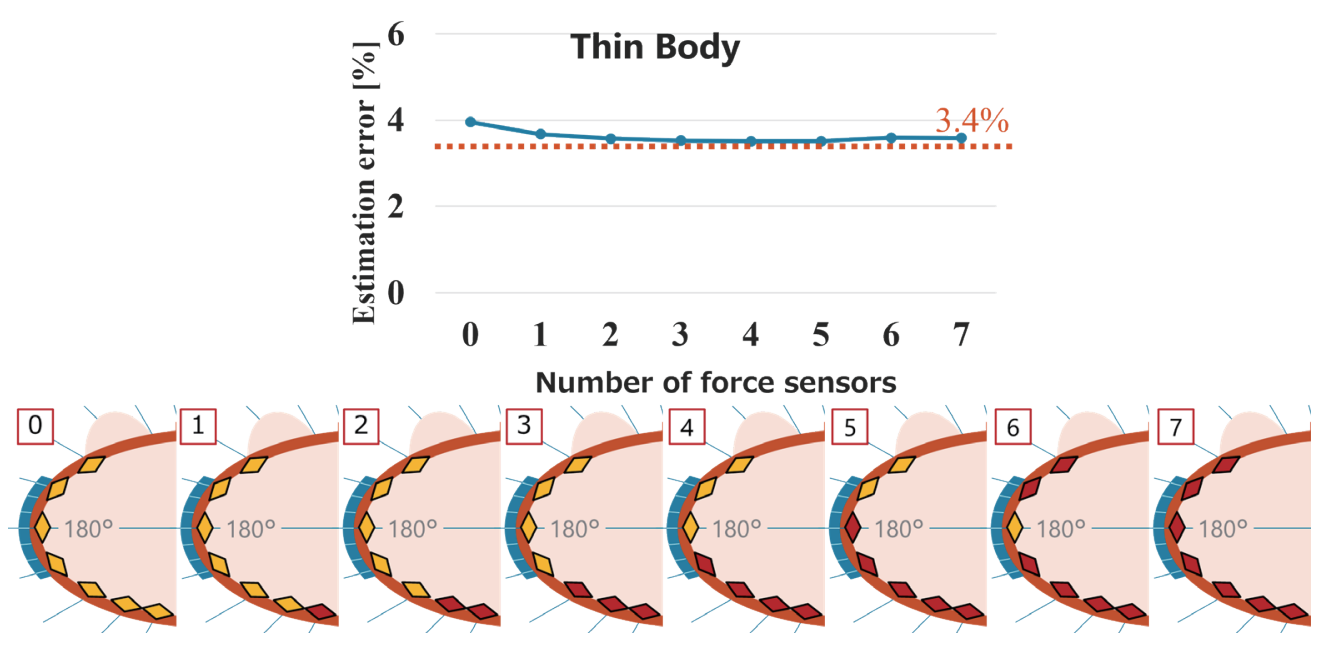

Fig. 11. (Color online) Average error of thin body vs number of force sensors.
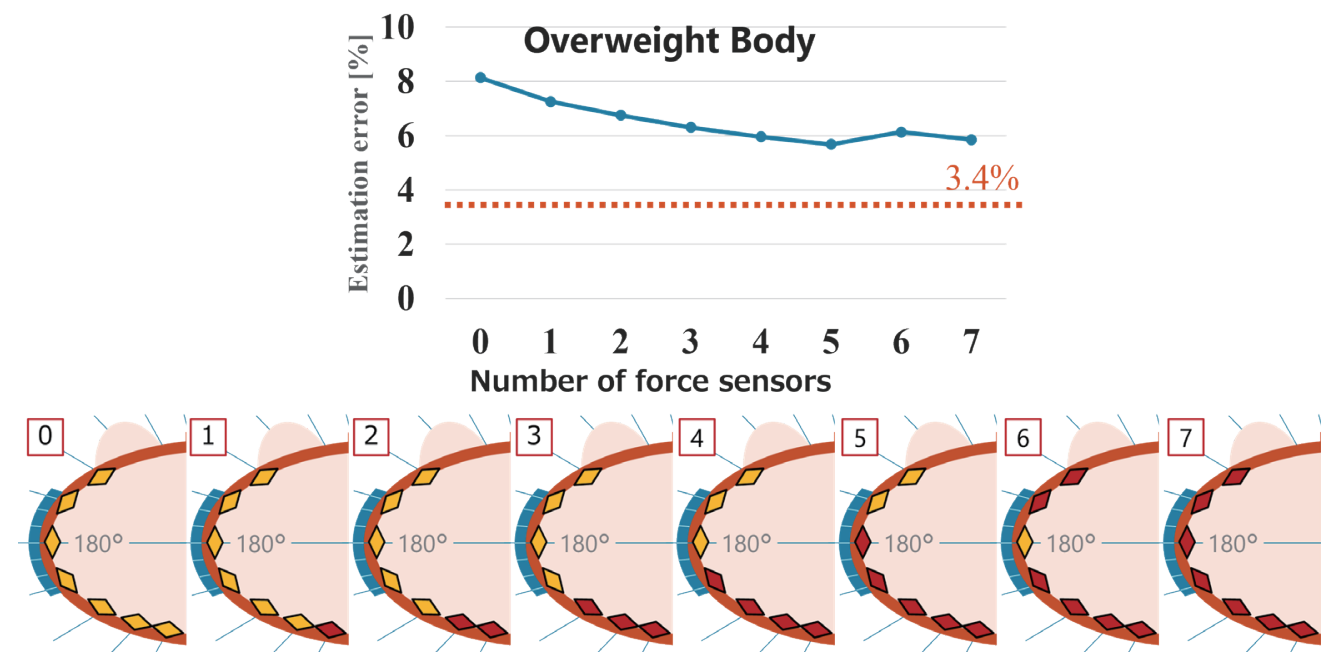

Fig. 12. (Color online) Average error of overweight body vs number of force sensors. 
the estimation error was reduced by using the force sensor at the back. This is because overweight subjects wear their belts more tightly, and the downward force $D_{F}$ due to the tightening is acquired by the force sensors, which we consider effective in estimating the abdominal circumference shape.

Figure 13 shows the results of estimating the belt shape $\left[D_{B}(\mathrm{ALL})\right]$ using the best combination of bending sensors for each body type and abdominal circumference shape $\left[D_{A}(\mathrm{ALL})\right]$ using all force sensors. Additionally, the measured values $\left(V_{M}\right)$ obtained with the measuring tool are

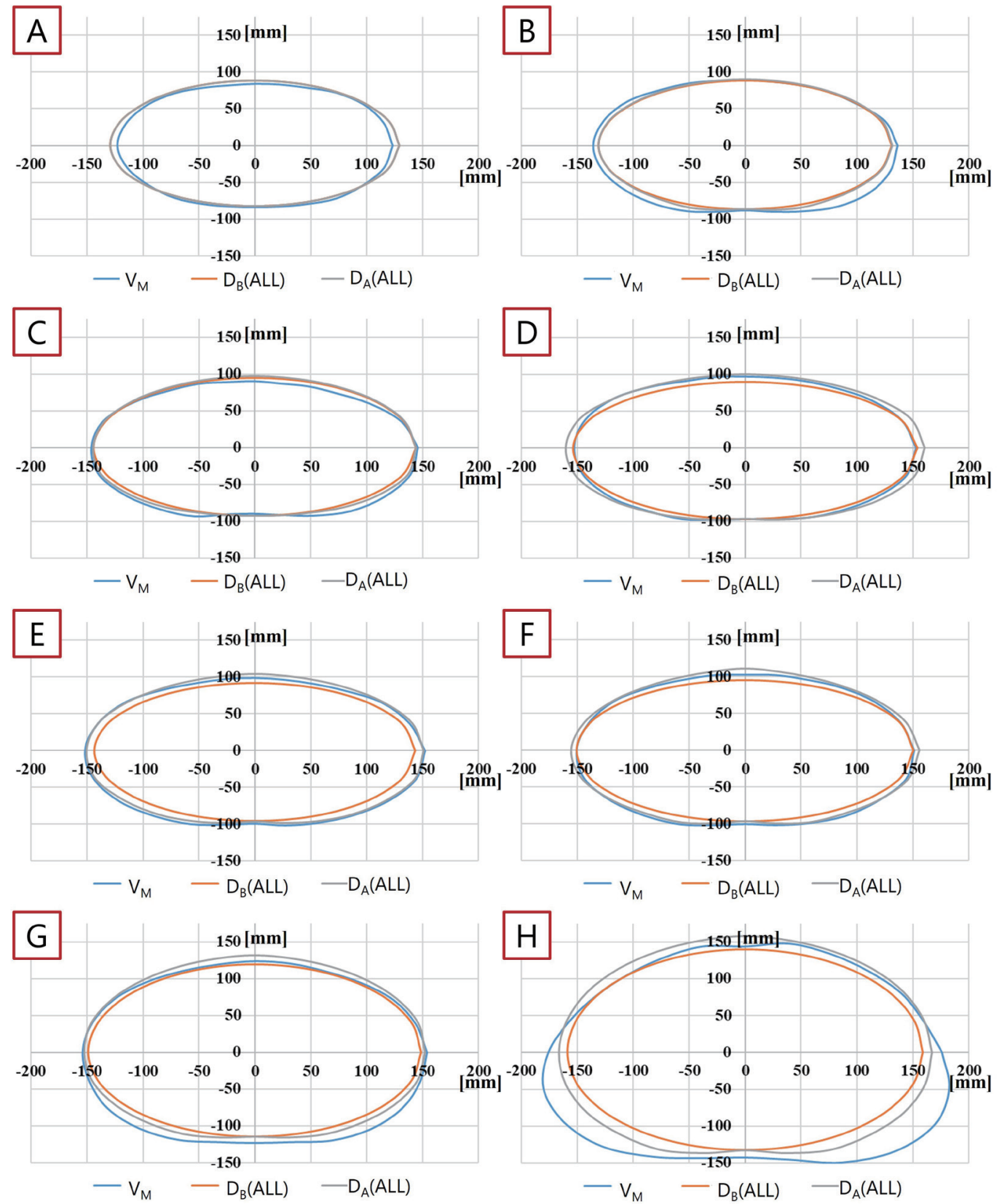

Fig. 13. (Color online) Results of abdominal circumference shape estimation using all force sensors. 
presented as the ground truth. There is no difference caused by the number of force sensors in the case of thin body type (A and B) compared with other body types. In contrast, the distribution of the force is added to the belt shape and the reference situation is estimated to be larger for overweight subjects $(\mathrm{G}$ and $\mathrm{H}$ ) than for thin subjects (A and $\mathrm{B}$ ). In the case of overweight subject $\mathrm{H}$, estimating the reference situation is difficult because the elliptical shape is different from the $V_{M}$ shape in Fig. 13. An accurate estimation of the belt shape should consider the approximation based on ellipse with different parameters.

\subsection{Optimal combinations of sensor positions per body type}

Figure 14 shows the combination of sensors with the smallest MAE. The sensor positions shown in the figure are considered effective for estimating the abdominal circumference and its shape for each body type. By using the best combination of sensors for each body type, we can estimate shapes with an average error of $3.73 \%$.

\section{Estimation of Reference Situation}

In the second experiment, we examine the algorithm to detect the timing close to the reference situation ${ }^{(21)}$ by comparing the changes in the shape of the abdominal circumference resulting from the differences in the situations.

\subsection{Method}

In this experiment, we acquired sensor data when the same subjects as in Sect. 4 performed the actions shown in Table 5 for $5 \mathrm{~s}$. These situations have state elements of posture, behavior,

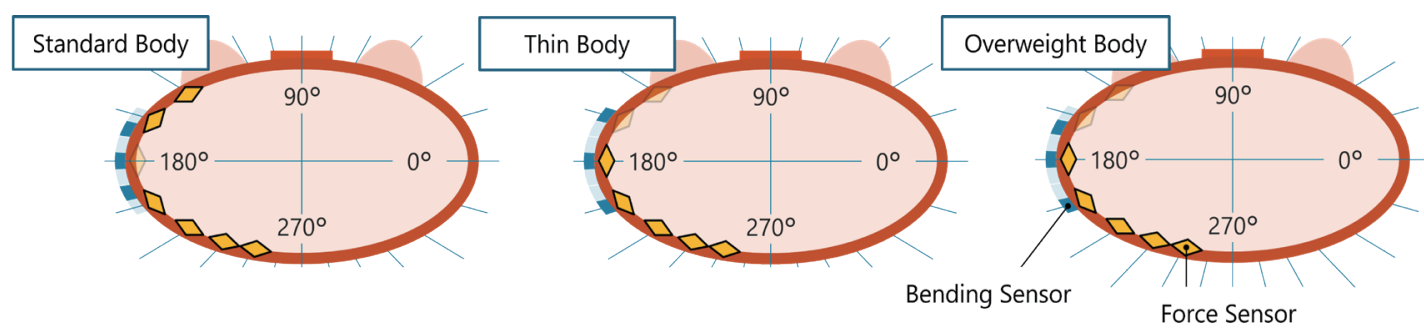

Fig. 14. (Color online) Optimal sensor positions.

Table 5

Situations of subject consisting of three state elements.

\begin{tabular}{lccc}
\hline Situation & Posture & Behavior & Respiration \\
\hline Reference & Standing & Static & Exhale \\
\hline Inspiratory & Standing & Static & Inspiratory \\
\hline Sitting & Sitting & Static & Exhale \\
\hline Standing in a slouch & Standing in a slouch & Static & Exhale \\
\hline Walking & Standing & Walking & Exhale \\
\hline
\end{tabular}


and respiration. The state combination of the reference situation includes standing, static, and exhale. Therefore, the different combinations of states are composed of inspiratory, sitting, standing in a slouch, and walking, because the posture, behavior, and respiration states changed compared with the reference situation. The shape of the abdominal circumference in each situation was estimated from the average of each sensor during the time period.

\subsection{Results and analysis}

First, we verify the detectability of the inspiratory state by comparing the inspiratory situation with the reference situation. Figure 15 shows two cases of abdominal circumference shapes in the reference situation and the inspiratory situation (subjects B and G) and indicates that the force sensor values increase in the front part, thus increasing the pressing pressure. The other six subjects showed similar tendencies. Additionally, we confirmed that the values of bending sensors did not change. In particular, $\mathrm{R}_{\mathrm{B}}$ [Eq.(5)] at $150^{\circ}$ was at least twice as large as the exhale state in the reference situation. This implies that the frontal force sensor at $150^{\circ}$ enables the detection of the reference situation.

The possibility of detecting the standing state is examined by comparing the reference situation (standing) with the sitting situation. Figure 16 shows two typical cases (subjects B and $\mathrm{H}$ ) of the comparison, in which the abdominal circumference was generally overestimated. In particular, the difference in the central part, i.e., $90^{\circ}$, was large. The other six subjects showed similar results. The values of bending sensors did not change between the standing and sitting states. Similar to the case of the comparison with the inspiratory situation, the value of $R_{B}$ in the reference situation at $150^{\circ}$ was at least twice as large as that of the sitting situation. Thus, we consider that the reference situation can also be detected by the frontal force sensor at $150^{\circ}$.

In the detection of another posture state, the reference situation (standing upright) and the "standing in a slouch" situation are compared. Figure 17 shows two examples (subjects D and F) of the comparison. The results of other subjects were the same. In the reference situation, the resistance of the force sensor in the front part was three times higher than that in the slouch state. Thus, we consider that it is also possible to detect the reference situation using the resistance of the frontal force sensor at $150^{\circ}$ to differentiate these situations.
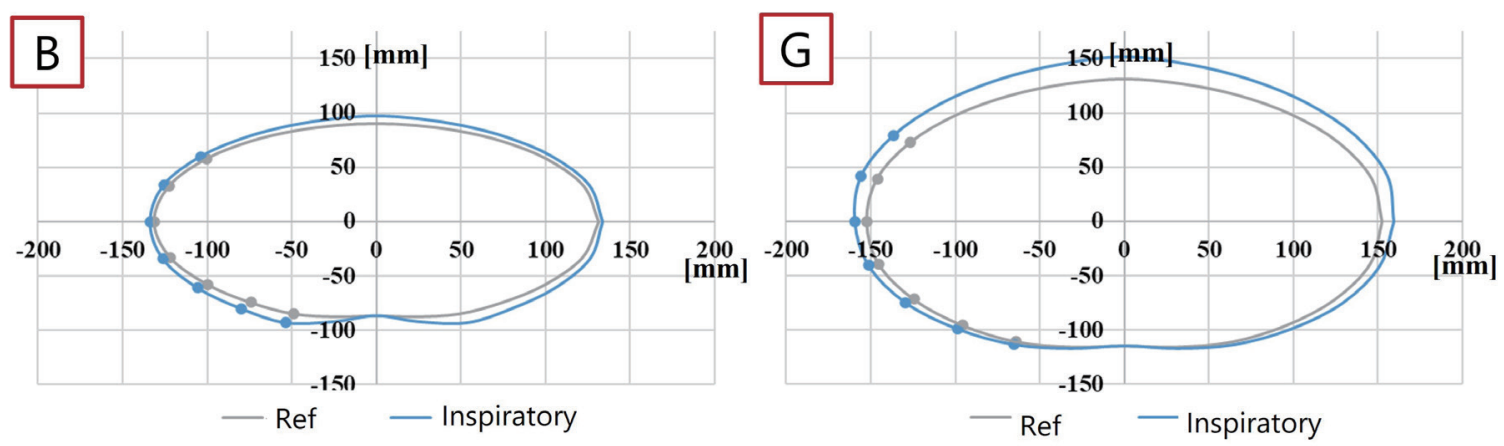

Fig. 15. (Color online) Differences between reference and inspiratory situations. 

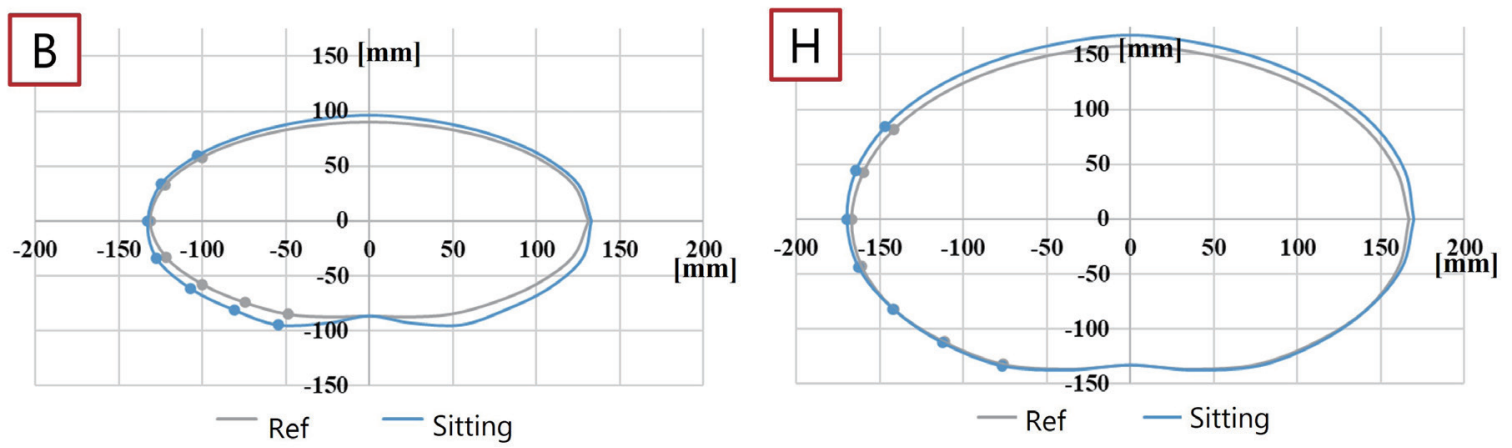

Fig. 16. (Color online) Differences between reference and sitting situations.
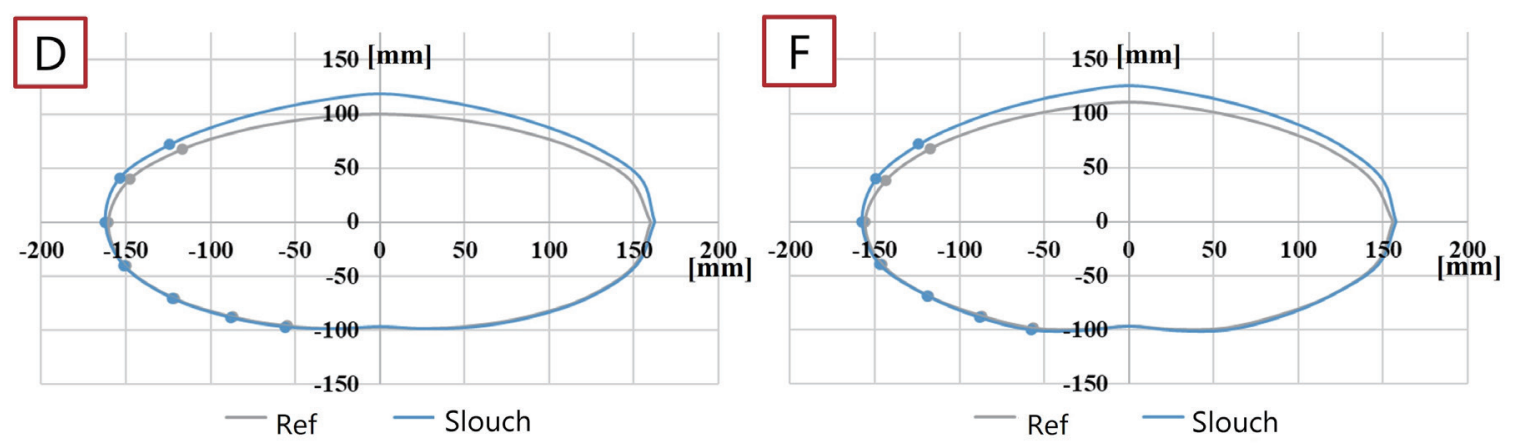

Fig. 17. (Color online) Differences between reference and standing in a slouch situations.

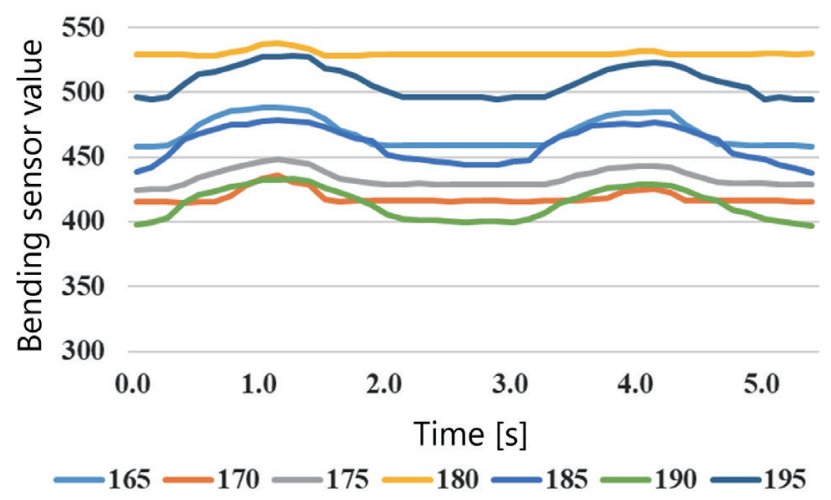

Fig. 18. (Color online) Bending sensor value during walking.

Figure 18 shows that the bending sensor value fluctuated when the wearer was walking. We consider that this fluctuation is caused by the force sensor being subjected to abdominal pressure due to walking. This sensor value fluctuation prevented the correct measurement of the radius of curvature using the bending sensor, which led to the failure of estimating the belt and abdominal circumference shapes. The result shows that the reference situation and the active, e.g., walking, situation can be discriminated by using the bending sensor values. The state is static if the bending sensor value is constant. 


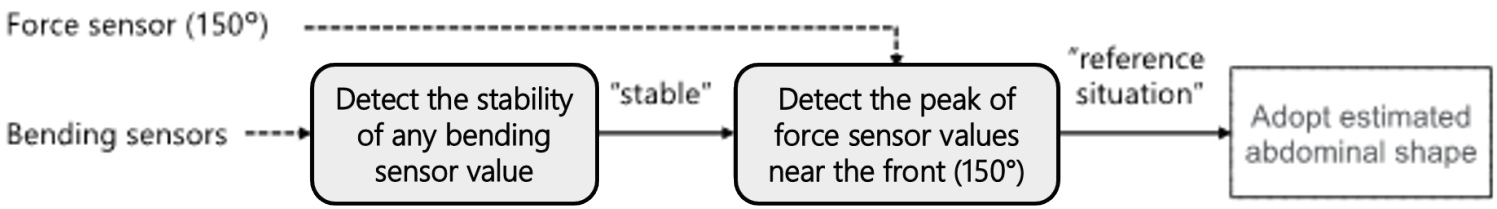

Fig. 19. Method of estimating reference situations.

\subsection{Towards the detection of reference situation}

From the analysis above, the procedure of detecting the reference situation is proposed as shown in Fig. 19. It consists of two processes: the detection of the stability of the bending sensor value and the detection of the peak of force sensor values. We assume that the reference situation and abdominal shape estimation processes run simultaneously. First, the stable period is detected to eliminate the period of the active state, e.g., walking, which is the flat period in Fig. 18, and we consider that any of the seven bending sensors can be used. Then, a peak moment at $150^{\circ}$ is detected in the values of the force sensor near the front. A large force sensor value indicates that the force applied to the sensor is small because the sensor value is inversely proportional to the applied force owing to the pull-down resistor as described in Sect. 3.3. Thus, the peak indicates that the belt is not pressed during the inspiratory, sitting, and slouch states. The peak moment represents the reference situation, and the abdominal shape estimated simultaneously is adopted. The implementation and evaluation are set for future work.

\section{Conclusions}

The objective of this study was to measure the shape of the abdominal circumference in a reference situation using a belt-type wearable device that can be worn daily. A prototype device consisting of seven bending sensors and seven force sensors on the belt was implemented. The bending sensors are used to estimate the belt shape by measuring the radius of curvature of the belt. The shape of the abdominal circumference was estimated by measuring the radius of curvature of the belt with a bending sensor. Also, by measuring the force applied to the belt with a force sensor, the abdominal circumference shape was observed to change with the tightening of the belt and, hence, the abdominal circumference without the belt was estimated.

We evaluated the appropriate combination of sensors for the estimation of the abdominal circumference shape with little error and compared the sensor values and the shape of the abdominal circumference in different states of the wearer. The experiment was conducted on eight subjects, and the abdominal measurement values obtained using the measuring tool and the abdominal circumference shape estimated using the proposed system were compared, and the estimation error was calculated. The results showed that it was possible to estimate the shape of the abdominal circumference with an average error of $3.73 \%$. As a result of comparing the sensor values in the reference situation and the dynamic state, it was clarified that the system could detect the upright and exhale states appropriate for measurement. 
In this study, the experiments were performed only on male subjects. In a study using MRI to measure visceral fat mass, ${ }^{(24)}$ the effective measurement position for estimating visceral fat mass in men was the waist, while it was the navel in females. In addition, females have less visceral fat than males, and estimating the force is considered to be challenging. Nevertheless, the contribution of this article is a novel method of estimating the shape of the abdominal circumference in daily life using a belt-type device. We believe that the proposed device can perform sufficient measurements, even for female users, by implementing methods such as changing the measurement position and the number of force sensors. In the future, we will develop a system adapted to females and improve the accuracy and miniaturization of the device, with the aim of improving the usability of the system.

\section{References}

1 National Health and Nutrition Examination Survey, Ministry of Health, Labor and Welfare: https://www.mhlw. go.jp/bunya/kenkou/kenkou eiyou chousa.html (accessed August 2021) (in Japanese).

2 C. Cercato and F. A. Fonseca: Diabetology Metab. Syndr. 11 (2017) 74. https://doi.org/10.1186/s13098-0190468-0

3 J. D. Tune, A. G. Goodwill, D. J Sassoon, and K. J. Mather: J. Lab. Clin. Med. 183 (2017) 57. https://doi. org/10.1016/j.trs1.2017.01.001

4 T. Miyawaki, M. Hirata K. Moriyama, Y. Sasaki, H. Aono, N. Saito, and K. Nakano: Proc. Japan Academy, Series B 81 (2005) 471. https://doi.org/10.2183/pjab.81.471

5 M. Nkwana, K. Monyeki, and S. Lebelo: Int. J. Environ. Res. Public Health 18 (2021) 281. https://doi. org/10.3390/ijerph18010281

6 A, Watanabe, S. Seno, H. Kobayashi, S. Kato, and H. Shimazu: Trans. Jpn. Soc. Med. Biol. Eng. 49 (2011) 156. (in Japanese).

7 K. Yamashita, T. Kikuchi, K. Masai, M. Sugimoto, B. Thomas, and Y. Sugiura. Proc. 23rd ACM Symp. Virtual Reality Software and Technology (ACM, 2017). https://doi.org/10.11184/his.20.3_311

8 J. M. Williams, I. Haq, and R. Y. Lee: Med. Eng. Phys. 32 (2010) 1043. https://doi.org/10.1016/j. medengphy.2010.07.005

9 L. E. Dunne, P. Walsh, S. Hermann, B. Smyth, and B. Caulfield: Trans. Biomed. Circ. Syst. 2 (2008) 97. https:// doi.org/10.1109/TBCAS.2008.927246

10 C. Chien, R. Liang, L. Lin, L. Chan, and B. Chen: Proc. 28th Annu. ACM Symp. User Interface Software \& Technology (ACM 2015) 659. https://doi.org/10.1145/2807442.2807456

11 K. Tsukada and M. Yasumura: Int. Conf. Ubi. Comp. (UbiComp, 2004) 384. https://doi.org/10.1007/978-3-54030119-6 23

12 R. Katzschmann, B. Araki, and D. L. Rus: Trans. Neu. Syst. Rehab. Eng. 1 (2018) 583. https://doi.org/10.1109/ TNSRE.2018.2800665

13 G. Cajamarca, I. Rodríguez, V. Herskovic, M. Campos, and J. C. Riofrío: Sensors 18 (2018) 3409. https://doi. org $/ 10.3390 / \mathrm{s} 18103409$

14 Y. Wu, R. Chen, and M. F. H. She: Software Engineering, Artificial Intelligence, Networking and Parallel/ Distributed Computing (Springer, 2011) p. 157. https://doi.org/10.1007/978-3-642-22288-7_13

15 Y. Matsuda, T. Hasegawa, I. Arai, Y. Arakawa, and K. Yasumoto: 9th Int. Conf. Mob. Comp. Ubi. Netw. (ICMU 2016) 1. https://doi.org/10.1109/ICMU.2016.7742089

16 B. Zhang, Y. Chen, H. Liu, and X. Wang: 13th Int. Conf. Ubi. Comp. (UbiComp, 2011) 473. https://doi. org/110.1145/2030112.2030177.

17 M, Funakoshi, K, Ikegawa, B. Shizuki, and S. Takahashi: SIG Hum. Comp. Interact. 34 (IPSJ, 2019) 1-6 (in Japanese).

18 W. Lee, E. Seto, K. Lin, and G. C. Migliaccio: Appl. Ergon. 65 (2017) 424. https://doi.org/10.1016/j. apergo.2017.03.016

19 T. Sztyler, H. Stuckenschmidt, and W. Petrich: Pervasive Mob. Comput. 38 (2017) 281. https://doi.org/10.1016/j. pmcj.2017.01.008

20 R. Fazio, D. Cafagna, G. Marcuccio, A. Minerba, and P. Visconti: Energies 13 (2020) 2161. https://doi. org/10.3390/en13092161 
1 S. Min, Y. Yun, and H. Shin: IEEE Sens. J. 14 (2014) 3245. https://doi.org/10.1109/JSEN.2014.2327991

22 Bending Sensor 55mm, https://www.switch-science.com/catalog/508/ (accessed August 2021) (in Japanese).

23 Force Sensor, https://www.switch-science.com/catalog/2613/ (accessed August 2021) (in Japanese).

24 Y. Takayanagi, S. Imamura, Y. Katayama, S. Muto, H. Oeda, and T. Masui: Ningen Dock 28 (2013) 622 (in Japanese).

\section{About the Authors}

Yukihiro Oishi received his B.S. and M.S. degrees in computer science from Tokyo University of Agriculture and Technology (TUAT), Japan, in 2019 and 2021, respectively. His research interests are in wearable and ubiquitous computing.

Airi Tsuji received her B.S., M.S., and Ph.D. degrees in engineering from Kyoto Institute of Technology, Japan, in 2010, 2012, and 2015, respectively. From 2015 to 2020, she was a researcher at the University of Tsukuba. Since 2020, she has been an assistant professor at TUAT. Her research interests are in human Interface, human-computer interaction, and welfare engineering. (atsuji@go.tuat.ac.jp).

Kaori Fujinami received his B.S. and M.S. degrees in electrical engineering and his Ph.D. degree in computer science from Waseda University, Japan, in 1993, 1995, and 2005, respectively. From 2005 to 2006, he was a visiting lecturer at Waseda University. From 2007 to 2017, he was an associate professor in the Department of Computer and Information Sciences at TUAT. Since 2018, he has been a professor at TUAT. His research interests are in machine learning, activity recognition, human-computer interaction, and ubiquitous computing. (fujinami@cc.tuat.ac.jp). 